\title{
CONTROL SOCIAL DEL TRÁFICO Y EMPODERAMIENTO SOCIAL. GRUPOS DE RIESGO: JÓVENES Y MAYORES
}

\author{
SOCIAL CONTROL OF TRAFFIC ACCIDENTS AND SOCIAL \\ EMPOWERMENT. RISK GROUPS: YOUNG PEOPLE AND OLDER PEOPLE
}

\section{Juan de Dios Izquierdo \\ Rubén Darío Torres Kumbrían \\ Laura Martínez}

Universidad Nacional de Educación a Distancia, Madrid. España/Spain

jizquierdo@,der.uned.es

rtorres@der.uned.es

laura.martinez@der.uned.es

Recibido/Received: 30/11/2012

Modificado/Modified: 16/09/2013

Aceptado/Accepted: 07/10/2013

\section{RESUMEN}

La evolución demográfica en Europa ha transformado la pirámide de edad y la pirámide de conductores hacia una pirámide invertida. Septuagenarios, octogenarios y nonagenarios inundan las carreteras, sin que se hayan adoptado las medidas de adecuación ergonómicas correspondientes. En el lado contrario, encontramos que la causa principal de muerte hasta los 34 años son los accidentes de tráfico, que se ceban con los jóvenes y niños. Se han puesto en marcha muchas medidas represivas que han dado resultados positivos. Pero hasta que los gobiernos no entiendan que es la sociedad, el control social del tráfico, el factor social, quien puede poner remedio, seguirá la pandemia.

\section{PALABRAS CLAVE}

Accidentes de tráfico, control social del tráfico, grupos de riesgo: mayores, jóvenes.

\section{SUMARIO}

1. Introducción. 2. Demografía y accidentes. 3. Mayores y jóvenes vulnerables en el tráfico. 4. Asimetría europea. 5. Hipótesis sobre la asimetría. 6. Conclusión. Bibliografía.

\begin{abstract}
The demographic evolution in Europe has transformed the drivers' age pyramid in a way that it is becoming the complete opposite of what it used to be. The roads are flooded with drivers aged 70-90 and no ergonomic measures have been taken to adapt the roads to this new situation. In addition, the main cause of death among the population of less than 34 years of age is road traffic accidents, mostly among teenagers and children. Although many punitive policies have been introduced and seem quite effective, governments need to acknowledge that only the social traffic control and the society itself are capable of putting an end to the accident pandemic.
\end{abstract}

\section{KEYWORDS}

Traffic accidents, social control of traffic, risk groups: young people and older people. 


\section{CONTENTS}

1. Introduction. 2. Demography and accidents. 3. Elderly and vulnerable young people in traffic. 4. European asymmetry. 5. Hypothesis about asymmetry. 6. Conclusions. References.

\section{INTRODUCCIÓN}

La Unión Europea suele hacer declaraciones y proyectos con mucho alcance estratégico. Normalmente, la distancia entre ellos y las medidas políticas que implementa es excesiva, si no abismal. En un espacio europeo del transporte, la política de seguridad vial está reservada a los Estados, sin que lo comunitario pase de recomendaciones, reuniones conjuntas de análisis y buenas declaraciones. Un ejemplo son las declaraciones de Antonio Tajani, entonces Comisario de Transportes, en una conferencia previa a la elaboración del Programa de acción europeo de seguridad vial 2011-2020:

"La consulta pública y la conferencia de hoy contribuyen a definir un programa de acción europeo de seguridad vial claro y mensurable para el período comprendido entre 2011 y 2020. Europa no puede ser laxista en seguridad vial, porque están en juego decenas de miles de vidas humanas cada año. Este Programa de acción nos ayudará a seguir siendo exigentes y decididos en nuestra lucha contra los accidentes de carretera. El nuevo Programa de acción también se centrará en prioridades clave tales como los accidentes en las carreteras rurales (que provocan el $60 \%$ del total de víctimas mortales) y los usuarios vulnerables de la vía pública, como los peatones, los ciclistas, los motoristas y los ancianos."

\section{DEMOGRAFÍA Y ACCIDENTES}

Es difícil entender que donde haya una pandemia no se vea concernido el trabajo social. En los ámbitos clásicos de la medicina y las enfermedades con sus secuelas, los servicios públicos puestos a disposición de los ciudadanos requieren de la colaboración de los trabajadores sociales. Antes y después de la asistencia sanitaria especializada, el trabajo social tiene una función inclusiva fundamental, porque el quirófano o la hospitalización no solventan la vulnerabilidad social que, a veces, en ciertos contextos socioeconómicos, la enfermedad implica.

Junto a los ámbitos tradicionales de la profesión, surgen otros a los que, sin metodologías todavía precisas, es necesario atender. Uno de ellos es el inmenso caudal de necesidad y sufrimiento que los accidentes de tráfico generan en la población. Sin olvidar la incidencia económica que la inseguridad vial ocasiona en las economías y que se cifra en un $2 \%$ del producto interior bruto europeo.

Frecuentemente, se asocia el trabajo social a momentos posteriores al problema social concreto de exclusión, pobreza, marginación, droga, violencia, casos de vulnerabilidad... Es lógico que se actúe ante hechos consolidados de necesidad social. No obstante, se impone, en la medida de lo posible, actuar preventivamente desde el análisis científico de las causas de la vulnerabilidad. En la materia que nos ocupa se diferencia entre seguridad vial pasiva, aquella que minimiza los daños cuando el accidente se produce, y seguridad vial activa, la que invierte en construir escenarios, elementos técnicos y formación que evite el accidente. El buen hacer de un médico, psicólogo o trabajador social puede lograr 
que un octogenario conductor, con derecho a conducir según la legislación europea, pero con mermas importantes en sus capacidades psicofísicas, deje voluntariamente de conducir. Es frecuente que su entorno familiar y social no consiga convencerle de que dejar el vehículo va en su propio beneficio, el de sus próximos, sus hijos y nietos, por cuya calidad de vida está dispuesto a hacer sacrificios.

Razones no le faltan, pues puede argumentar que no ha tenido accidentes ni multas en los últimos meses o años, dada la prudencia con que conduce y las medidas de precaución que adopta: evitar las horas punta, no conducir de noche, mantener una velocidad moderada, no recorrer grandes distancias, etc. Posiblemente sus hijos no puedan rebatirle, pero saben que su visión no es suficiente para los grandes requerimientos que el tráfíco actual requiere, que sus movimientos se han lentificado progresivamente, que sus reflejos no son los adecuados, que los adelantamientos que efectúa son peligrosos.

Todos estos argumentos serían contundentes con cualquier pequeña cifra de personas mayores surcando nuestras carreteras, pero adquiere una dimensión radical cuando observamos la figura de evolución demográfica en Europa o elegimos un país para ajustar la proyección (Gráfico 1).

GRÁFICO 1: Proyección de la pirámide de conductores
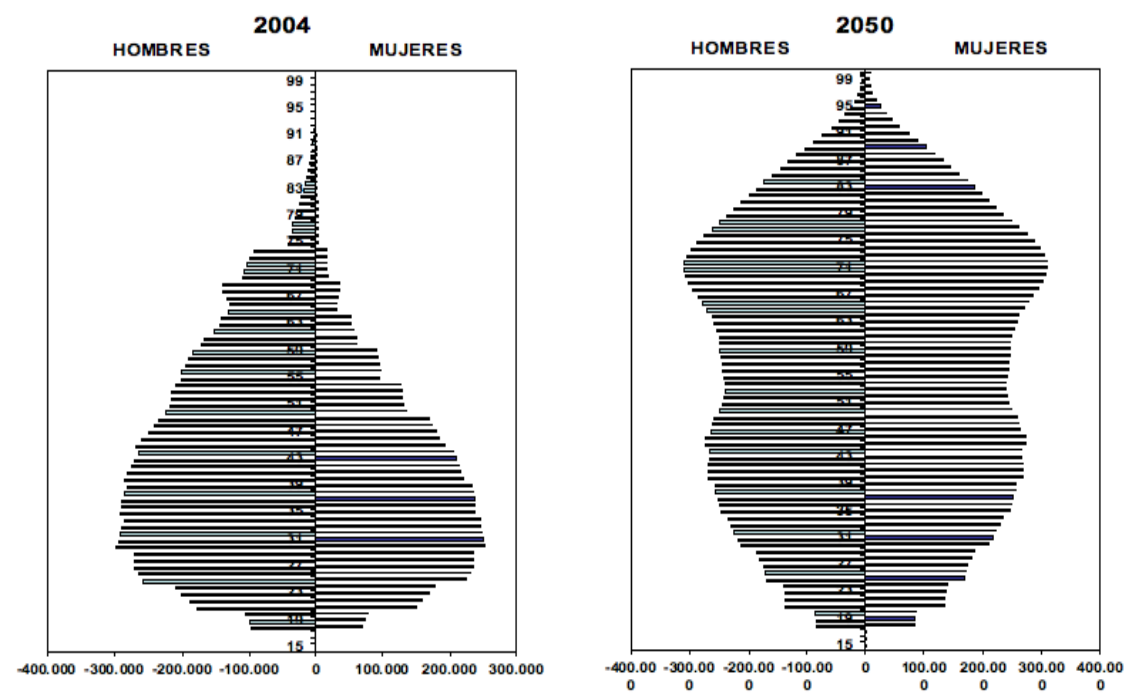

Fuente: UNESPA

Los mayores están ocupando las cohortes más amplias de la pirámide y transformando con gran rapidez la situación hasta invertirla. Independientemente de la asimetría entre hombres y mujeres que se aprecia en la pirámide de 2004, propia de un país europeo que llega tarde a la promoción social de la mujer en Europa, la proyección de 2050 nos presenta ante un panorama de millones de europeos octogenarios y nonagenarios inundando las carreteras secundarias, las autovías y las autopistas, que se han socializado con el vehículo privado, en el que se sienten seguros y sin el que se encuentran inermes, inútiles y dependientes. 
Posiblemente, la inmigración europea cambie parcialmente esta imagen de una Europa envejecida, porque la población inmigrante introduce tasas de natalidad superiores, que traducidas en el $10 \%$ de la población que alcanza en cada vez más países, puede alterar la fisonomía de la pirámide de población y, consecuentemente de conductores. Además de conductores, los mayores tienen el rol de acompañantes en los vehículos, -cuando no conducen-, y de peatones. En ambos casos su situación contribuye de forma importante a la pandemia de víctimas mortales o heridos.

\section{MAYORES Y JÓVENES VULNERABLES EN EL TRÁFICO}

Las muertes de personas mayores en los contextos urbanos no decrecen en la medida de las expectativas de las políticas públicas y de los planes de reducción del $50 \%$ del Libro Blanco del Transporte Europeo (Gráfico 2), (CE 2001).

GRÁFICO 2: Número de peatones mayores de 49 años heridos graves en zona urbana

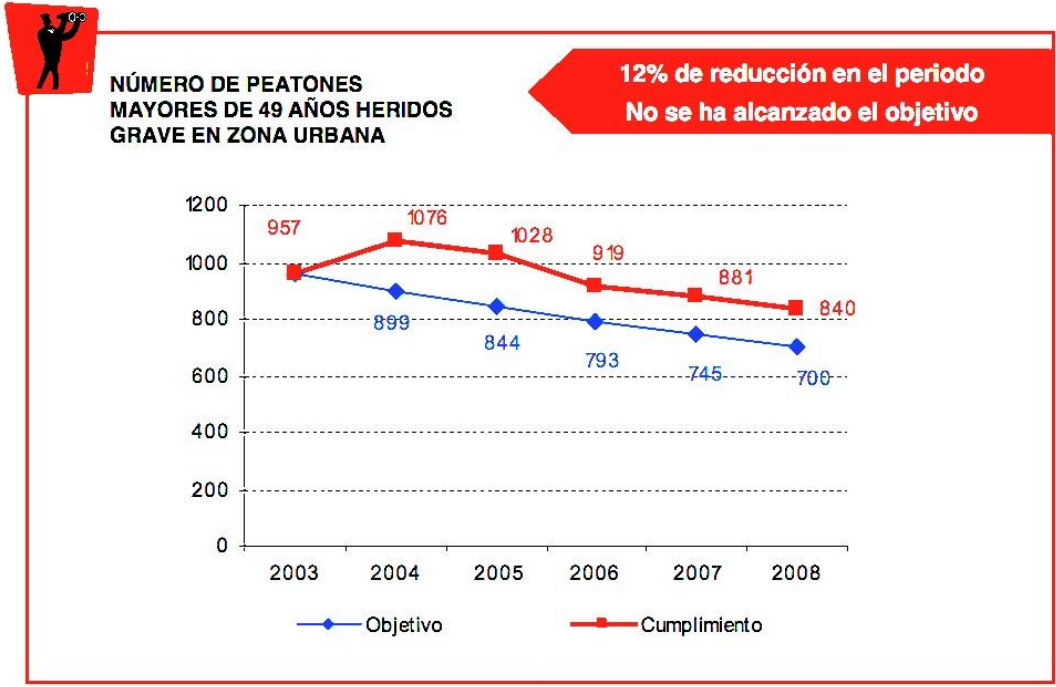

Fuente: DGT 2009

Son varias las causas que merecen atención, con el fin de corregir la tendencia y mejorar la seguridad de nuestros mayores. El análisis no es inocuo porque de su acierto depende la reducción de los muertos y heridos que en número inadmisible se producen.

El colectivo más remiso a la reducción de accidentes perseguido como objetivo prioritario por el "Programa de acción europeo de seguridad vial" son los mayores. Incluso el sector de jóvenes está reaccionando de una forma estadística mejor, aunque es cierto que partía de porcentajes exageradamente altos.

Podríamos ya avanzar que la ergonomía de todos los ingredientes que intervienen en la seguridad vial no se adapta a las condiciones y los requerimientos objetivos de un colectivo inmenso, que se está convirtiendo en mayoritario. Los vehículos, señalizaciones, vías, servicios, normativa etc., no están incorporando las adaptaciones necesarias para 
evitar la accidentalidad de conductores, peatones y acompañantes de los mayores. Por no hablar de la precarización del transporte profesional (Izquierdo y López 2011).

\section{ASIMETRÍA EUROPEA}

Los datos de los distintos países europeos nos permiten comparar la accidentalidad entre ellos y a establecer hipótesis sobre las sustanciales diferencias que se dieron y aún persisten entre países del norte de Europa y países del sur. Para fortalecer el contraste, exponemos resultados de años en que la diferencia era más llamativa que en los últimos ejercicios.

Es cierto que la variable de fallecidos por millón de habitantes no es la más analítica que se puede encontrar porque no mide la movilidad de los ciudadanos. Si no es utilizado el vehículo en la misma proporción entre los referentes comparados, estaría viciada sustancialmente la correlación. Pero merece ser visualizada la diferente tasa de mortalidad entre los primeros (menor mortalidad) y los últimos de este ranking, al ser tan desproporcionada que ninguna matización analítica puede minimizarla.

Entre los 38, 39, 39 y 52 muertos por millón de habitantes de Reino Unido, Holanda, Suecia y Finlandia y los 95, 118, 129 y 79 de Luxemburgo, Bélgica, Grecia y Portugal existe una sima tan profunda que deben buscarse causas también profundas (Gráfico 3).

GRÁFICO 3: Fallecidos por millón de habitantes 2000-2009 (UE 27)

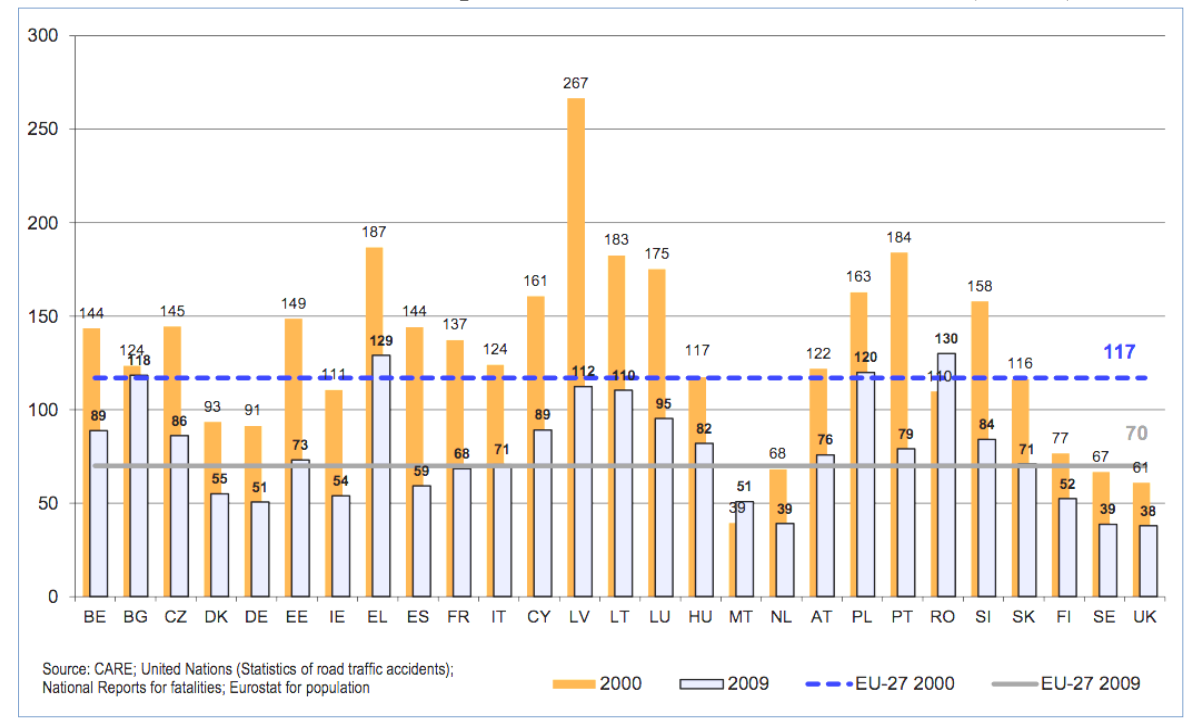

Fuente: Eurostat 2011

Antes de pasar a ponderar las distintas hipótesis, merece la pena establecer la comparación mediante los factores de movilidad a los que aludíamos, con el fin de desestimar posibles diferencias de nivel de utilización del vehículo y de kilómetros de movilidad por habitante. Existen países en que la oferta y la cultura de utilización de los transportes públicos es netamente superior a otros. Nos referimos a la oferta en cuanto a 
infraestructuras (estaciones, paradas, perímetro de alcance en los transportes públicos de cercanías a las ciudades, flujos y regularidad de los mismos, nivel de confort de autobuses y trenes). Nos referimos a la cultura de utilización del transporte público, no como tradición solamente, sino como priorización política del mismo frente al vehículo privado, preservación del medio ambiente, tasas de circulación por el centro de las ciudades, dificultad de aparcamientos, creación de plazas gratuitas o baratas de aparcamiento del vehículo privado en las entradas de las ciudades, para que el usuario pueda utilizar el transporte público dentro del contexto urbano etc. Estas diferencias de movilidad en los distintos modos afecta a la accidentalidad, además de a la congestión y la contaminación.

El nuevo parámetro recoge una menor dispersión, pero deja en evidencia el caso de Grecia que con una menor movilidad parece pertenecer a otra galaxia analítica, especialmente si se concentra la comparación con los cuatro países modelo.

Estos análisis y las tasas correspondientes de accidentalidad, con todos sus efectos están en la base de decisiones políticas importantes que se han venido tomando por la Unión con el fin de reducir la sangría inaceptable en vidas, heridos y costes correspondientes.

\section{HIPÓTESIS SOBRE LA ASIMETRÍA}

La asimetría europea expuesta crece con la ampliación a 27 miembros y se genera el chequeo de hipótesis que pueden estar operando como variables eficaces de la misma:

* Según el clásico Tri-level Study (Treta, 1977) los factores ligados al estado de las carreteras, incluidos los medioambientales, ejercen una influencia escasa o moderada en los accidentes de tráfico. En el 12\% es considerado factor clave y en el $34 \%$ concurrente. Estudios recientes han seguido manteniendo la estela de factor secundario adjudicado a las infraestructuras. Es discutible, especialmente cuando no se considera únicamente el firme, el peralte en las curvas o la señalización, sino el desdoblamiento de calzada con el consiguiente ahorro de los adelantamientos frontales. Pero en el caso de nuestros datos comparativos es digno de reseñar que tanto Luxemburgo como Bélgica, con vías en excelente estado de conservación y desdoblamiento, se encontraban entre los países más inseguros de los analizados. No puede recaer la carga de la prueba de la asimetría de la inseguridad en las infraestructuras viarias.

* Otro factor a examinar es el nivel de los vehículos. Otro clásico (NHTSA 1987) reduce la incidencia prioritaria del vehículo en los accidentes a un 9\%, incluyendo los concausados. En la UE, no todos los países poseen el mismo parque automovilístico desde el prisma de la seguridad. Las gamas altas están mejor equipadas de instrumentos de seguridad pasiva y activa. Pero los países descritos se caracterizaban por poseer buenos vehículos y mala seguridad. Estudios posteriores, no siempre financiados por las empresas constructoras de automóviles, siguen sosteniendo que el vehículo no es la causa.

* El conductor (el factor humano) se perfila como el gran responsable de la accidentalidad (entre el 70 y el $90 \%$ de la causa principal) y es sectorializado para su estudio preciso:

1. Aptitud: adaptarse a la máquina fue contemplado en cierta época como causa importante. Hoy está descartado. Dominar técnicamente la máquina no se presenta como problema. 
2. Conocimiento: sobre la vía, el vehículo y sus prestaciones, la normativa. Los ejercicios para obtener el carné de conducir están impregnados de este convencimiento. Los accidentes no discriminan entre conductores con mayor o menor conocimiento.

3. Actitud: el campo de la psicología científica viene trabajando sobre este vector y logrando (cursos de rehabilitación de conductores que han perdido el carné por puntos) que se considere muy importante la actitud que debe llevar el conductor al volante.

Es evidente que la aptitud, los conocimientos y la actitud son factores de base que deben estar perfilados y valorados en el modelo típico-ideal weberiano del buen conductor. Pero ¿son suficientes para comprender y evitar la siniestralidad vial? Parece que no, a tenor de la alta accidentalidad que padecemos.

El psicologismo que se ha expandido alrededor del factor humano aporta ingredientes positivos para captar sistémicamente el problema. El error es reducir a actitud y a factores psicológicos la profundidad del mismo. Si no se es consciente de que el factor humano, tal y como es contemplado por algunos investigadores, es una reificación inadecuada (Parsons) estaremos convirtiéndolo más en una parte del problema que de la solución. La interacción social es la clave de la accidentalidad evitable: la relación del conductor con los otros conductores, peatones, usuarios de la vía y normativa que lo ordena. Más aún con los valores sociales y la prioridad que la sociedad en general concede a un buen comportamiento vial. El comportamiento del conductor en relación con esa interacción social es la clave de la accidentalidad, no la relación del conductor con su conciencia y con su actitud. Estamos hablando del factor social. Las sociedades cuya cultura, es decir, cuyos valores, cuyo compromiso, no minusvalora la gravedad de una conducción insegura, tomará medidas. No sólo legales, sino sociales, de indignación, rechazo, manifestación de desacuerdo, cuando un conductor pone en peligro por velocidad, agresividad, inconsciencia, falta de respeto de la norma a los ciudadanos. Esta tarea en los países del sur de la Unión está delegada en la policía, en los controles y en una normativa crecientemente dura, tanto a nivel económico como jurídico (se puede ir a la cárcel si víctimas por medio). Este sistema sancionador tiene su eficacia. La reducción de la accidentalidad, importante, que se ha producido en los países de peores ratios, se debe casi por completo a la dureza de la norma y a la capacidad de control (Tabla 1).

España ha cambiado de metabolismo en materia de control y normativa, siendo el país de los quince analizados que mejor comportamiento evidencia. No se puede minusvalorar los avances de Luxemburgo (-50\%), Francia, Portugal, entre los países que manifestaban peor comportamiento. Tampoco el de aquellos que partiendo de una buena posición han mejorado aún en porcentajes considerables (Reino Unido, Holanda, Suecia). Peor evolución encontramos en Grecia y Bélgica.

Efectivamente, la presunción, por parte del conductor, de ser controlado en velocidad alcoholemia, drogas, cinturón de seguridad, casco, le lleva a comportamientos acordes con la norma. Pero es difícil mantener esa actitud una vez que el peligro ha pasado y se vuelve al modo de conducción habitual (Gráfico 4).

Los gobiernos son pragmáticos y acuden a aquellas medidas que de forma inmediata ofrecen resultados tangibles. En este caso, además, la legitimación es plena porque el saldo supone un ahorro de víctimas mortales y heridos tan importante que ninguna instancia generará una crítica alternativa. Si además se consigue cumplir las demandas europeas de reducción del $50 \%$ que demandaba el Libro Blanco del Transporte, el éxito político complementario refuerza tanto las medidas como las orientaciones estratégicas que se han adoptado. 
TABLA 1: La siniestralidad en Europa

\begin{tabular}{|c|c|c|c|c|c|c|c|}
\hline & 2001 & 2007 & 2008 & 2009 & $2009-2008$ & $2008-2007$ & $2009-2001$ \\
\hline Bélgica & 1.486 & 1.071 & 944 & 655 & $-30,6 \%$ & $-11,9 \%$ & $-55,9 \%$ \\
\hline Letonia & 558 & 419 & 316 & 254 & $-19,6 \%$ & $-24,6 \%$ & $-54,5 \%$ \\
\hline España & 5.517 & 3.823 & 3.100 & 2.605 & $-16,0 \%$ & $-18,9 \%$ & $-52,8 \%$ \\
\hline Estonia & 199 & 196 & 132 & 100 & $-24,2 \%$ & $-32,7 \%$ & $-49,7 \%$ \\
\hline Portugal & 1.670 & 974 & 885 & 840 & $-5,1 \%$ & $-9,1 \%$ & $-49,7 \%$ \\
\hline Francia & 8.162 & 4.620 & 4.275 & 4.273 & $0,0 \%$ & $-7,5 \%$ & $-47,6 \%$ \\
\hline Lituania & 706 & 739 & 499 & 370 & $-25,9 \%$ & $-32,5 \%$ & $-47,6 \%$ \\
\hline Eslovaquia & 614 & 627 & 558 & 347 & $-37,8 \%$ & $-11,0 \%$ & $-43,5 \%$ \\
\hline Italia & 7.096 & 5.131 & 4.731 & 4.050 & $-14,4 \%$ & $-7,8 \%$ & $-42,9 \%$ \\
\hline Irlanda & 412 & 338 & 279 & 240 & $-14,0 \%$ & $-17,5 \%$ & $-41,7 \%$ \\
\hline Alemania & 6.977 & 5.056 & 4.477 & 4.152 & $-7,3 \%$ & $-11,5 \%$ & $-40,5 \%$ \\
\hline Suecia & 583 & 471 & 397 & 355 & $-10,6 \%$ & $-15,7 \%$ & $-39,1 \%$ \\
\hline Esolvenia & 278 & 293 & 214 & 171 & $-20,1 \%$ & $-27,0 \%$ & $-38,5 \%$ \\
\hline Holanda & 993 & 709 & 677 & 644 & $-4,9 \%$ & $-4,5 \%$ & $-35,1 \%$ \\
\hline Finlandia & 433 & 380 & 344 & 281 & $-18,3 \%$ & $-9,5 \%$ & $-35,1 \%$ \\
\hline Reino Unido & 3.598 & 3.059 & 2.645 & 2.337 & $-11,6 \%$ & $-13,5 \%$ & $-35,0 \%$ \\
\hline Austria & 958 & 691 & 679 & 633 & $-6,8 \%$ & $-1,7 \%$ & $-33,9 \%$ \\
\hline Luxemburgo & 70 & 43 & 35 & 47 & $34,3 \%$ & $-18,6 \%$ & $-32,9 \%$ \\
\hline República Checa & 1.334 & 1.221 & 1.076 & 901 & $-16,3 \%$ & $-11,9 \%$ & $-32,5 \%$ \\
\hline Dinamarca & 431 & 406 & 406 & 306 & $-24,6 \%$ & $0,0 \%$ & $-29,0 \%$ \\
\hline Hungria & 1.239 & 1.232 & 996 & 882 & $-11,4 \%$ & $-19,2 \%$ & $-28,8 \%$ \\
\hline Chipre & 98 & 89 & 82 & 71 & $-13,4 \%$ & $-7,9 \%$ & $-27,6 \%$ \\
\hline Grecia & 1.880 & 1.612 & 1.555 & 1.453 & $-6,6 \%$ & $-3,5 \%$ & $-22,7 \%$ \\
\hline Polonia & 5.534 & 5.583 & 5.437 & 4.572 & $-15,9 \%$ & $-2,6 \%$ & $-17,4 \%$ \\
\hline Bulgaria & 1.011 & 1.006 & 1.061 & 901 & $-15,1 \%$ & $5,5 \%$ & $-10,9 \%$ \\
\hline Rumania & 2.450 & 2.800 & 3.061 & 2.796 & $-8,7 \%$ & $9,3 \%$ & $14,1 \%$ \\
\hline Malta & 16 & 12 & 15 & 21 & $40,0 \%$ & $25,0 \%$ & $31,3 \%$ \\
\hline Unión Europea & 54.303 & 42.601 & 38.876 & 34.257 & $-11,9 \%$ & $-\mathbf{- 8 , 7} \%$ & $-36,9 \%$ \\
\hline & & & & -6 & & \\
\hline
\end{tabular}

Fuente: RACC e IRTAD 2011

El carné por puntos y las disposiciones legales de cárcel han operado, junto con los radares fijos y móviles en la disminución de la velocidad media, lo que inmediatamente tiene efectos de reducción de víctimas.

GRÁFICO 4: \% de Conductores sancionados durante los últimos tres años comparados con los que esperan que controlen su velocidad

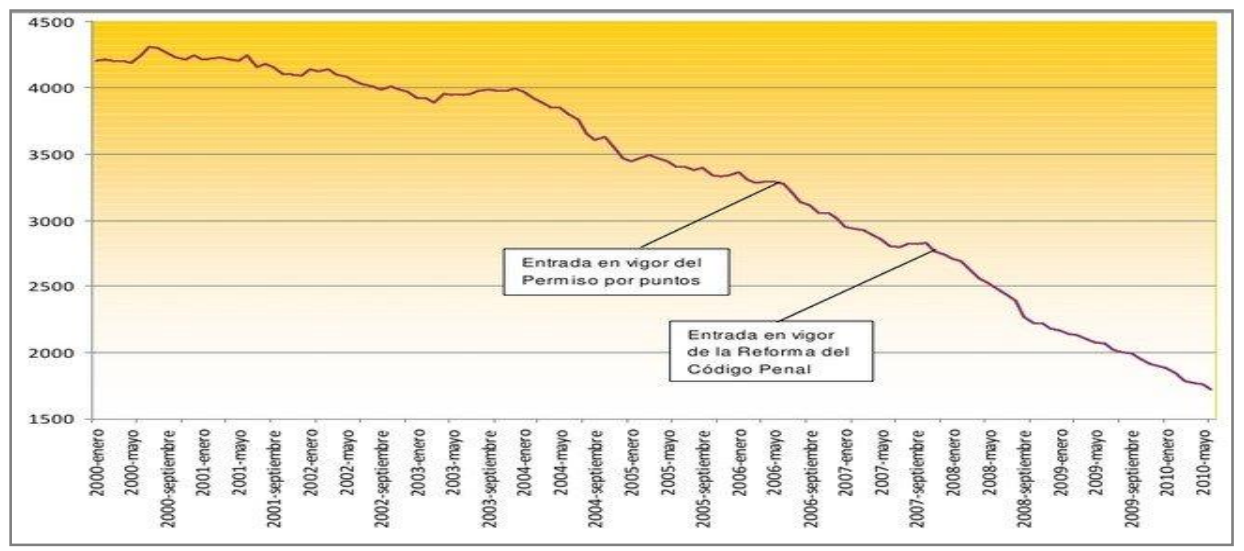

Fuente: RACC 2010 
Si apreciamos el gráfico siguiente (Gráfico 5), podemos comprobar que el descenso es espectacular, aunque el descenso venía precedido por otras medidas (controles, radares, aumento de la cuantía de las multas).

GRÁFICO 5: Carnet por puntos y víctimas mortales

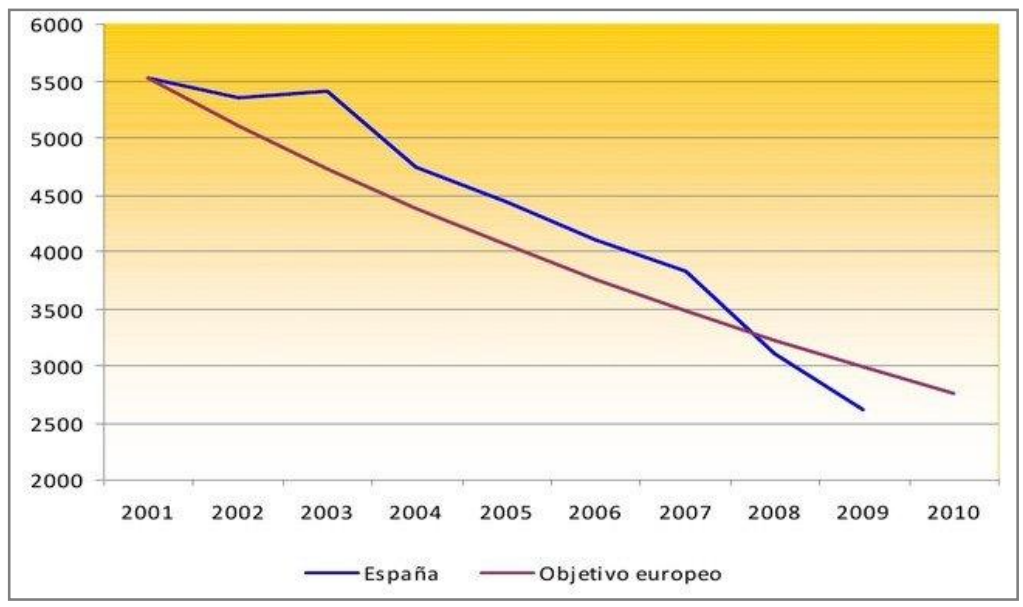

Fuente: RACC 2010 FITSA (2004). Seguridad vial. Barómetro 2004. Madrid

En las reuniones de coordinación de los responsables de seguridad de los distintos países europeos, favorecidas por la Unión, comienza, sin embargo a cundir la preocupación porque los límites de las medidas coercitivas adoptadas hasta ahora comienzan a ser visibles.

GRÁFICO 6: Objetivo de reducción del 50\% de víctimas mortales

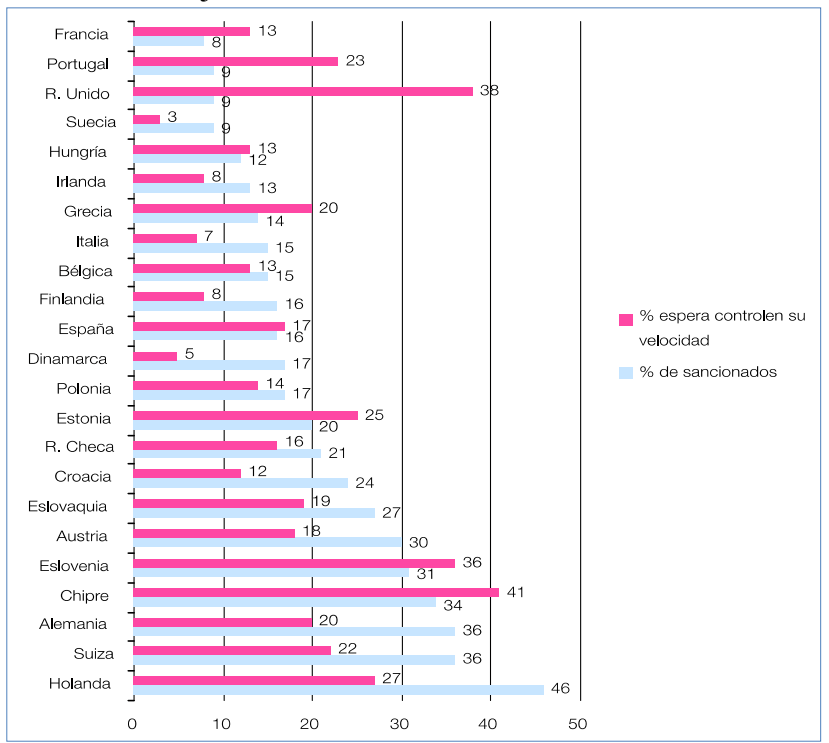

Fuente: RACC 2010 
En bastantes países, las cifras de siniestralidad ya no son tan fácilmente comparables con las del año anterior. No pocos fines de semana el resultado es peor que en el mismo periodo del año anterior, aunque la propaganda gubernamental siga haciéndose eco de la histórica reducción del 50\% entre el 2001 y el 2010. Es preciso valorar lo que el gráfico siguiente indica: 5.500 víctimas mortales en 2001 y 2.600 en 2009 (Gráfico 6).

Como comentábamos más arriba, los mayores de 65 años se han convertido en el sector poblacional que menos ha reducido sus víctimas. Esto requiere una reflexión que vaya más allá de la mera contemplación estadística, si queremos aportar vías de solución al efecto.

Un dato inesperado ha sido la buena dirección marcada por los jóvenes, con porcentajes elevadísimos de disminución. Sigue siendo un sector de riesgo en seguridad vial, pero es indudable que las medidas generales, los cambios producidos en las infraestructuras en relación con las motos, los controles de alcoholemia y droga, además de las campañas a favor de que conduzca el miembro del grupo que está de guardia, sobrio, han incidido en su mejora.

Especialmente reseñable es la disminución de más del $60 \%$ de la accidentalidad en el doble sector que va de los 15 a los 34 años, líder en víctimas (Gráfico 7).

El rol que cumple el alcohol en la relación entre jóvenes es importante y se tiende a descomponerlo analíticamente con el fin de poder atacar el consumo inadecuado, especialmente ligado a la conducción.

GRÁFICO 7: Víctimas mortales en carretera por grupos de edad

\begin{tabular}{|c|c|c|c|}
\hline Grupos de edad & 2010 & $\begin{array}{c}\text { Comparativa } \% \\
2010 / 2003\end{array}$ & $\begin{array}{c}\text { Comparativa } \% \\
2010 / 2003\end{array}$ \\
\hline Hasta 14 años & 49 & $-53 \%$ & $-53 \%$ \\
\hline 15 a 24 & 259 & $-68 \%$ & $-68 \%$ \\
\hline 25 a 34 & 319 & $-66 \%$ & $-63 \%$ \\
\hline 35 a 44 & 317 & $-51 \%$ & $-53 \%$ \\
\hline 45 a 54 & 256 & $-49 \%$ & $-53 \%$ \\
\hline 55 a 64 & 194 & $-48 \%$ & $-52 \%$ \\
\hline+ de 65 & 318 & $-43 \%$ & $-46 \%$ \\
\hline
\end{tabular}

Fuente: DGT 2011 y elaboración propia

Los estudios SARTRE (SARTRE, 2012) demuestran que no existe una conciencia asumida del peligro del alcohol, exceptuando la situación de embriaguez evidente. El carácter de mediador cultural que la ingesta de alcohol tiene en las sociedades europeas tiende a minusvalorar los graves efectos de una ingesta que no inhabilita para el desarrollo de otros trabajos. En los jóvenes, el consumo de alcohol incorpora otros elementos de importancia, relacionados con la desinhibición en las relaciones de grupo, de pareja, de aproximación y facilitación de trato mutuo, en una etapa de la evolución psicológica con especiales dificultades de formación.

Mayores y jóvenes, con plena capacidad para los roles de conductores, peatones y acompañantes, necesitan, dada su morbilidad en materia de seguridad vial, tratamiento diferenciado para incidir con profundidad en sus respectivas problemáticas (Gráfico 8). Existe una base analítica suficiente, aunque no excesiva de su problemática. Con ella se pueden llevar a cabo actuaciones, desde los distintos ámbitos institucionales, públicos, fundacionales o privados para avanzar propuestas intersectoriales que contribuyan a reducir drásticamente los efectos letales que para una familia supone la pérdida de la vida, la tetraplejia, la incapacidad de un joven, un mayor, una persona. 


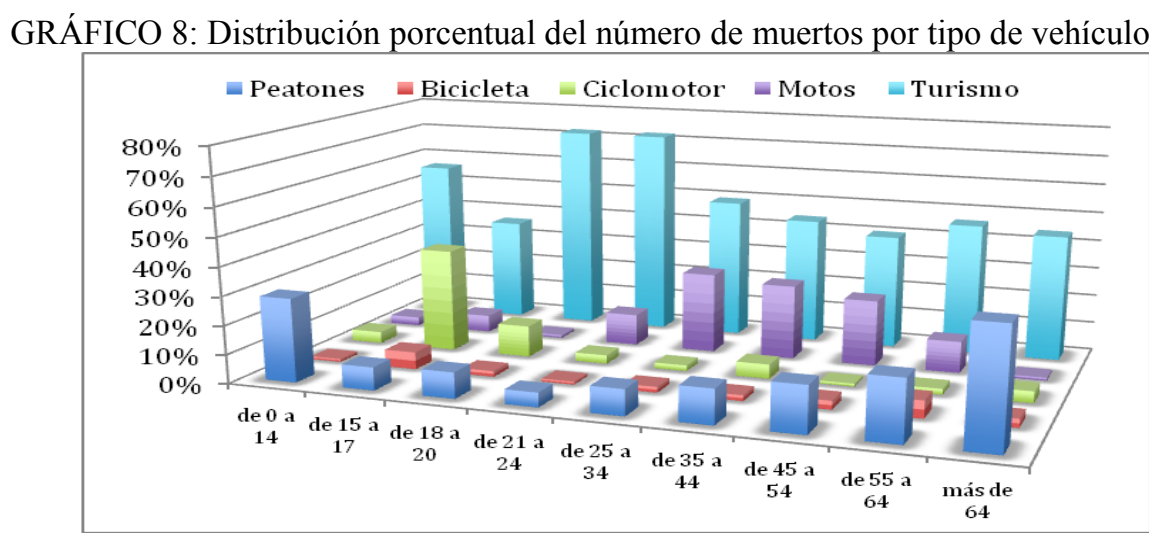

Fuente: DGT 2010 y elaboración propia

\section{CONCLUSIONES: EL TRABAJO SOCIAL ANTE COLECTIVOS VULNERABLES EN LA VÍA PÚBLICA}

Las instituciones públicas, en los últimos años (DGT 2009), están llevando a cabo estudios (sondeos, investigaciones, análisis de resultados de las medidas propuestas en anteriores planes). Algo similar ocurre con otras instituciones fundacionales 0 aseguradoras. En estos proyectos suelen estar presentes psicólogos profesionales, médicos, ingenieros, algún sociólogo. Ni politólogos ni trabajadores sociales han participado, como profesionales con prismas sinérgicos necesarios.

Las conclusiones del estudio de la DGT de 2009, ya hace referencia a la importancia del médico (en el grupo de trabajo de personas mayores) como profesional de contacto con el colectivo. Se considera que tiene credibilidad suficiente para recomendar a las personas mayores dejar de conducir por limitaciones funcionales. Sin objetar la gran labor que los profesionales de la medicina pueden aportar desde el ejercicio de su profesión, podemos preguntarnos cuál es, y especialmente, cuál debe ser el rol del trabajador social en esta problemática. Para responder a esta cuestión es preciso analizar dos factores previos: a) qué nivel de conocimiento, contacto y credibilidad tiene el trabajador social en los dos sectores analizados (mayores y jóvenes); b) qué nivel de investigación está desarrollando el trabajo social en la investigación de estos y otros sectores en los que tiene un bien acreditado trabajo de campo.

Parece evidente que si comparamos el contacto de los trabajadores sociales con mayores y jóvenes con el que tienen otros sectores profesionales los primeros acreditan mayor continuidad que ningún otro. El trato permanente de los trabajadores sociales en residencias, hogares, asociaciones, barrios, con seguimientos de casos, familias, comunidades, con estos colectivos es elevado. La calidad de su relación y la credibilidad que los trabajadores sociales acreditan es alta. No se trata de un momento en una consulta médica o psicológica, sino, en la mayoría de los casos, de una relación continuada, intermediadora, amistosa. Este caudal y capital relacional que los trabajadores sociales poseen les puede convertir en interlocutores privilegiados a la hora de llevar a cabo políticas destinadas a colectivos vulnerables para evitar su vulnerabilidad. Es, pues, 
necesario reclamar el espacio que se ocupa como intercambiador social, por parte de la profesión, para contribuir a la mejora de la situación en el tema que nos ocupa.

En segundo lugar, es preciso ser autocríticos con la realidad objetiva de la investigación en los ámbitos sociales en que se mueven los profesionales del trabajo social. Estando en una posición inmejorable, por la condición de observación participante, los trabajadores sociales han cumplido el rol de agentes con información cualificada a los que los sociólogos y psicólogos han sometido a encuestas cualitativas de investigaciones, pocas, en cuyo diseño no han participado, no conocen y de cuyas conclusiones no tienen información y mucho menos control.

Además de demandar participación plena en proyectos que necesariamente deben ser interdisciplinares, cuando administración o empresas efectúen investigaciones, se requiere una demanda creciente de canteras y proyectos concretos en los que la investigación social es necesaria para avanzar. Conviene poner en valor a los investigadores y a las revistas de trabajo social y de ciencias sociales que están abriendo posibilidades a los investigadores para exponer sus resultados, en los ámbitos clásicos del trabajo social y en los nuevos emergentes, a los que se debe estar atentos y definir, conceptualizar, estudiar para acercarnos a ellos con la mayor documentación y profundidad posible. En este sentido, la aproximación que planteamos ante el problema de la accidentalidad en el tráfico y sus secuelas es el apuntado como factor social. Ni la calidad de las vías, ni el nivel tecnológico del parque automovilístico, ni los conocimientos de los conductores, ni la actitud de los mismos es la clave para acercarnos a la solución posible del problema de la accidentalidad.

La diferencia extraordinaria en número de muertes o heridos en los distintos países europeos tiene, a nuestro juicio, una base social no técnica. Las sociedades que han sabido convertir el comportamiento vial correcto en una prioridad, con lo que implica, han obtenido resultados y se han implicado en ellos. Las que no, han delegado la solución de la pandemia a los funcionarios de tráfico. Ante la inaceptable cantidad de muertes por accidentes de trafico (1.300.000 accidentes, 35.000 muertes, 1.700 .000 heridos, anualmente, en la Unión Europea), el desconcierto y la perplejidad produce el bloqueo social. El doble error de que "el accidente es fortuito" y que la envergadura del mismo requiere un tratamiento complejo en el que deben intervenir sólo los especialistas produce la inhibición y el bloqueo social.

Ejemplos como la acción contra la violencia de género, la defraudación fiscal o la pederastia muestran que, si se dotan de prioridad social, si la sociedad los incorpora como valores urgentes e importantes en su acervo cultural, pueden, en muy escaso tiempo, producir un cambio de metabolismo positivo. Construir esa capacidad individual, grupal, colectiva, social, en relación con un objetivo, es dotar de mecanismos, expresión y capacidad de actuación, de empoderamiento.

La aportación de los trabajadores sociales a este empoderamiento no puede basarse sólo en motivaciones altruistas, sino que requiere, como profesionales, un equipamiento analítico y crítico de cara a sus objetivos. Aunque el objetivo estratégico podamos expresarlo como "trasladar a la sociedad y la administración que se debe asumir con realismo, tanto en su dimensión demográfica como en sus circunstancias específicas, el fenómeno del envejecimiento y su incidencia en el tráfico", estamos obligados a descomponer analítica y propositivamente ese mensaje estratégico.

La pirámide de edad de los conductores europeos se está transfigurando con gran rapidez. Pero los vehículos, la normativa, las carreteras, los controles, los avances tecnológicos, las señales, siguen teniendo como referente al tipo-ideal de conductor y automóvil de la otra pirámide desfasada: joven, con capacidad adquisitiva, amante de la 
velocidad y el confort, complejo, infectado de tecnología que nunca utilizará, exhibicionista. Frente a esta oferta para jóvenes, el contacto con los mayores aporta datos a tener en cuenta para confeccionar vehículos, vías, información y control. Los millones de europeos mayores de 65 años tienen auténtica adicción a su vehículo, con el que han convivido más de 40 años, en el que se encuentran seguros, a pesar de haber sufrido algún accidente.

Para demandar la ergonomía integral adaptada a la nueva pirámide de conductores y estar en condiciones de reclamar a los mayores autocontrol para dejar de conducir, es preciso conocer que el apego al vehículo no es algo superficial, que se puede solventar con una conversación. Para una persona de más de 65 años el automóvil supone independencia, libertad de movimientos, utilidad para solventar sus demandas de compras y relaciones, disponibilidad para la familia, acceso al lugar de término sin estar sometido a sus dificultades motoras. Si a ello sumamos que los medios públicos de transporte no siempre están próximos ni son fácilmente accesibles el apego al vehículo es justificado.

La sensación de jubilación en el sentido negativo del término está, en no pocos casos ligado más a la necesidad de abandonar el carné que a la de cumplir la etapa laboral. La sensación de dependencia, de ser una carga, de inutilidad para colaborar con la familia, son sentimientos profundos que inciden en la pereza a dejar de conducir.

Por ello, deben valorarse los motivos que convierten en una colaboración razonable con la familia del mayor, la recomendación profesional: problemas de memoria, atención, percepción de la complejidad de detalles que acompañan a la conducción, la exigencia de decisiones que deben tomarse en tiempo mínimo, los problemas motores, artritis, enfermedades propias de los mayores y las medicaciones correspondientes, que en muchos casos llevan aparejadas efectos de somnolencia, lentificación de reflejos y otras. A todo ello debe sumarse el plus de vulnerabilidad que los mayores tienen ante accidentes similares +3.5 .

La contribución al empoderamiento social requiere conocer científicamente los factores que se reclaman el estado objetivo del sector desde el que se trabaja y la participación correspondiente, sin delegar en expertos.

El factor social como clave para la solución de la inseguridad vial, del que hemos avanzado algunos aspectos, requiere de un Pacto de Estado que clarifique el carácter prioritario que la sociedad concede a la cultura vial. Esto es una condición fundamental para que el comportamiento vial no se solvente sólo entre conductor y aparato normativo, sino entre el conductor y los peatones u otros conductores que son testigos de su forma de conducir (Tabla 2).

Cada sociedad establece la forma de conducir que admite. Así, el conductor observa la reacción que su conducción genera en los demás actores con los que interactúa. De esta forma, el conductor está recibiendo una auditoría externa permanente, que le exige estar por encima de los posibles problemas que le acompañan, de su actitud, de su humor. Si no se comporta conforme al modelo, al límite social, recibirá recriminaciones permanentes.

Existen estudios de comunidades europeas que se han instalado en países distintos del suyo y se han adaptado a los hábitos y exigencias en la conducción de la sociedad receptora.

No se debe minimizar la importancia que los accidentes de tráfico ejercen sobre la cohesión social. El impacto en las familias pobres puede generar procesos de desestructuración fulminantes (Izquierdo y Torres, 2011) 
Participar en las investigaciones, estudiar los casos en que los mayores deben plantearse dejar de conducir, aunque legalmente puedan seguir haciéndolo, analizar situaciones de comportamiento de los jóvenes en relación con el tráfico e influir en ellos para reconducir su actitud, son prácticas de tratamiento preventivo sobre sectores vulnerables que merecen ser tratados con profesionalidad.

TABLA 2: Modelo de Pacto de Estado para la Seguridad Vial

\begin{tabular}{|c|}
\hline Pacto político \\
\hline $\begin{array}{l}\text { - Prioridad politica } \\
\text { - Prioridad presupeustaria } \\
\text { - Prioridad investiga dora } \\
\text { - Prioridad participativa }\end{array}$ \\
\hline Pacto social \\
\hline $\begin{array}{l}\text { - Prioridad laboral } \\
\text { - Cultura de empresa en seguridad vial } \\
\text { - Concertación }\end{array}$ \\
\hline Pacto de participación asociativa \\
\hline $\begin{array}{l}\text { - Asociac. de transporte de viajeros } \\
\text { - Asociac. de transprore de mercancía } \\
\text { - Constructor es de infraestructuras } \\
\text { - Constructor es de vehículos } \\
\text { - Centros de investigaciones de seguridad vial } \\
\text { - Asociac. de Seguridad vial } \\
\text { - Asociac. de vecinos (peatones) } \\
\text { - Asociac. de vehículos } \\
\text { - Asociac. de donantes de sangrey órganos } \\
\text { - Medios de comunicación }\end{array}$ \\
\hline Pacto institucional \\
\hline $\begin{array}{l}\text { - Administraciones públicas } \\
\text { - Federación de Municipios y Provincias } \\
\text { - DGT } \\
\text { - Agencia Española de Seguridad Vial para la carr etera }\end{array}$ \\
\hline
\end{tabular}

Fuente: Elaboración propia

La continuidad de los trabajadores sociales en la comunicación con estos colectivos les confiere unas características y valores que no pueden aportar otros profesionales cuyo contacto con ellos es puntual y en circunstancias de crisis. Los efectos de ese vínculo facilita la labor de empowerment.

\section{BIBLIOGRAFÍA}

COMISIÓN EUROPEA (1999), Hacia una Europa para todas las edades. COM (1999) 221 final. Bruselas.

COMISIÓN EUROPEA (2001), Libro Blanco. La política europea de transportes de cara al 2010: la hora de la verdad. COM 2001/370 FINAL). Bruselas. 
COMISIÓN EUROPEA (2002), La respuesta de Europa al envejecimiento a escala mundial. Promover el progreso económico y social en un mundo en proceso de envejecimiento. Contribución de la Comisión Europea a la segunda Asamblea Mundial sobre el Envejecimiento. COM (2002) 143 final. Bruselas.

COMISIÓN EUROPEA (2003), Programa de acción europeo de seguridad vial. Reducir a la mitad el número de víctimas de accidentes de tráfico en la Unión Europea de aquí a 2010: una responsabilidad compartida COM (2003) 311 final. Comunicación de la Comisión. Bruselas.

COMISIÓN EUROPEA (2004), Programa de Acción Europeo de Seguridad Vial. Bruselas.

CONSEJO EUROPEO DEL TRANSPORTE (2001). Report on transport and ageing of the population. European Conference of Ministers of Transport, Council of Ministers. París.

CONSEJO EUROPEO (2000), Decisión 2000/750 del Consejo, de 27 de noviembre de 2000, por la que se establece un programa de acción comunitario para luchar contra la discriminación (2001-2006). París.

DGT (2004), SARTRE 3. Actitudes sociales frente al riesgo vial en Europa. Resultados de la muestra española. Dirección General del Tráfico del Ministerio del Interior. Madrid.

DGT (2009), Plan Estratégico de Seguridad Vial 2010-2015 de la Dirección General de Tráfico del Ministerio del Interior. Madrid.

ELVIK, R. (1997), "The effects on accidents of automatic speed enjorcement in Norway", (Paper no 970018), Tranportation Research Board, 76th Annual Meeting. Washington D.C.

ELVIK, R. y TRULS, V. (2006), The Handbook of Road Safety Measures, Amsterdam, Elsevier. FITSA (2004). Seguridad vial. Barómetro 2004. Madrid.

HAKAMTES-BLOMQVÍST, L. (2003), Ageing Europe: the challenges and opportunities for transport safety [5a Conferencia sobre la seguridad de los transportes en Europa], Bruselas, European Transport Safety Council.

IZQUIERDO, J. y LÓPEZ, A. (2011), “La construcción social de la precariedad laboral: paradojas de la liberalización del transporte terrestre en España". Revista de Sociología del Trabajo. No 73, pp.60-79.

IZQUIERDO, J.; MUÑOZ, A. y TORRES, R. (2006), Diagnóstico y Evaluación Social de los Accidentes de Tráfico. Madrid, Fundación UNED.

IZQUIERDO, J. y TORRES, R. (2006), Nueva Cultura Sistémica de la Seguridad Vial: Hacia el Control Social del Tráfico. Hacia una Seguridad Sostenible: Una estrategia para todos, Madrid, Asociación Española de la Carretera.

IZQUIERDO, J. y TORRES, R. (2007), "Movilidad, dependencia y transporte. Nuevas demandas de la población mayor en España. Dependencia y Transporte", La protección de las personas mayores, Madrid, Tecnos, pp. 451-462.

IZQUIERDO, J. y TORRES, R. (2010a), "El colectivo de Mayores, los accidentes de tráfico y el Trabajo Social" en Portularia, 1, Huelva, pp. 33-49.

IZQUIERDO, J. y TORRES, R. (2010b), El control Social del tráfico, Madrid, Fundación Francisco Corell.

IZQUIERDO, J. y TORRES, R. (2011), "Cohesión euroamericana (2011). Supranacionalidad de los derechos sociales frente a la ortodoxia neoliberal" Revista de Derecho Social, no 61, pp. 20-45

MONTORO, L. et al. (1995), Seguridad Vial: del factor humano a las nuevas tecnologías, Madrid, Síntesis.

MONTORO, L. et al. (2000), Manual de Seguridad Vial: El factor humano, Ariel.

NOVAK, J. W y R. P. SHUMATE, R.P. (1961), "The use of 'control groups' in highway accidentresearch: A field study". Traffic safety Research Review, pp. 20-24.

NHTSA (National Highway Traffic Safety Administration), (1998), Traffic Safety Facts 1997, U.S. Department of Transportation, Washington.

OMS (2004a), Carta de los derechos fundamentales de los mayores, aprobada en Niza el 7 de diciembre de 2000.

OMS (2004 b), Primer Plan de Acción Internacional sobre Envejecimiento (2002), Naciones Unidas, Asamblea Mundial sobre envejecimiento, Madrid.

OMS (2004c), Informe mundial sobre prevención de traumatismos causados por el tránsito. OMS, Washington DC. 
SARTRE Consortium (2012), European drivers and road risk. INRETS, Arcueil.

TREAT, J. R. et al. (1977), Tri-level study of the causes off traffic accidents. Bloomington, Indiana, Indiana University.

\section{Breve currículo:}

\section{Juan de Dios Izquierdo}

Profesor Titular, Director del Departamento de Trabajo Social (UNED), Investigador Invitado del CADIS, París. Premio a la Investigación otorgado por la Fundación Corell. Líneas de investigación: Estado de Bienestar, Cohesión Económica y Social Europea, Género. Profesor de Máster y Doctorado en Problemas Sociales.

\section{Rubén Darío Torres Kumbrían}

Profesor Contratado Doctor (UNED), e Investigador Invitado de la Universidad de Varsovia. Premio a la Investigación Fundación Francisco Corell. A sus dos tesis doctorales relativas a las mujeres musulmanas y a la cohesión social europea se deben añadir publicaciones sobre Género, Cohesión y Estado del Bienestar.

\section{Laura Martínez}

Profesora Ayudante (UNED), Licenciada en Humanidades, Máster en Trabajo Social. Publicaciones sobre el impacto de las TIC y las redes en el ámbito profesional. Líneas de investigación: Estado de Bienestar, Tecnologías de la Información y la Comunicación, Impacto de las redes sociales. 